\title{
MOTION ARTIFACT REDUCTION IN FUNCTIONAL NEAR INFRARED SPECTROSCOPY SIGNALS BY AUTOREGRESSIVE MOVING AVERAGE MODELING BASED KALMAN FILTERING
}

\author{
MEHDI AMIAN* and S. KAMALEDIN SETAREHDAN \\ Control and Intelligent Processing Centre of Excellence \\ School of Electrical and Computer Engineering, College of Engineering \\ University of Tehran, Tehran, Iran \\ *mehdi.amian@alumni.ut.ac.ir
}

Received 30 June 2013

Accepted 3 August 2013

Published 4 September 2013

\begin{abstract}
Functional near infrared spectroscopy (fNIRS) is a technique that is used for noninvasive measurement of the oxyhemoglobin ( $\mathrm{HbO} 2)$ and deoxyhemoglobin $(\mathrm{HHb})$ concentrations in the brain tissue. Since the ratio of the concentration of these two agents is correlated with the neuronal activity, fNIRS can be used for the monitoring and quantifying the cortical activity. The portability of fNIRS makes it a good candidate for studies involving subject's movement. The fNIRS measurements, however, are sensitive to artifacts generated by subject's head motion. This makes fNIRS signals less effective in such applications. In this paper, the autoregressive moving average (ARMA) modeling of the fNIRS signal is proposed for state-space representation of the signal which is then fed to the Kalman filter for estimating the motionless signal from motion corrupted signal. Results are compared to the autoregressive model (AR) based approach, which has been done previously, and show that the ARMA models outperform AR models. We attribute it to the richer structure, containing more terms indeed, of ARMA than AR. We show that the signal to noise ratio (SNR) is about $2 \mathrm{~dB}$ higher for ARMA based method.
\end{abstract}

Keywords: Brain; Gaussian noise; linear model; state estimation.

\section{Introduction}

Functional near infrared spectroscopy (fNIRS) is a relatively recent technique for noninvasive measurement of oxygenated hemoglobin (oxy-Hb) and deoxygenated hemoglobin (deoxy-Hb) concentrations in the human brain. ${ }^{1}$ Other applications include quality control, pharmacology and medical diagnoses. $^{2}$ Basically, a typical fNIRS system is composed

*Corresponding author.

This is an Open Access article published by World Scientific Publishing Company. It is distributed under the terms of the Creative Commons Attribution 3.0 (CC-BY) License. Further distribution of this work is permitted, provided the original work is properly cited. 
of one or a number of light sources in near infrared (NIR) range (700-900 $\mathrm{nm}$ ), and several detectors that collect the reflected photons from the brain tissue. In this spectrum, water and vital tissues are relatively transparent and by investigating the collected light intensities (fNIRS signals), the properties of the medium through which the light has passed can be identified. ${ }^{2,3}$

This technique is affordable, portable and capable of being used in real field applications., ${ }^{2,4,5}$ Also, fNIRS is safe compared to other imaging techniques such as $\mathrm{X}$ ray imaging, positron emission tomography (PET), nuclear medicine and computed tomography (CT). As such, it has been widely used for studies with vulnerable populations such as neonates. However, such applications entail inevitable head movements. As the head motion can increase the blood flow through the scalp and rarely causes an increase in brain's blood pressure, ${ }^{2}$ therefore, motion artifact may change original signal and lead to incorrect result or misguided diagnose.

Reducing head motion artifacts is therefore a key aspect in signal processing area of fNIRS studies and there have been a number of attempts. Adaptive filtering is used as one of the main artifact removal methods. ${ }^{6}$ The Wiener filter was also applied to the fNIRS signals. ${ }^{7}$ Another method to reduce so called "head motion noise" in fNIRS signals has been developed on the principle that there is a negative correlation between oxygenated and deoxygenated haemoglobin concentration changes. ${ }^{8}$ A method based on moving standard deviation and spline interpolation enables semi-automatic detection and reduction of motion artifact in near infrared imaging signals. ${ }^{9} \mathrm{~A}$ wavelet based approach was used by Molavi et al. ${ }^{10}$ Although these along with other methods could reduce the motion artifact from fNIRS signals but each method has some specific requirements and limitations. For example, in the adaptive filter based method, ${ }^{6}$ the algorithm needs additional hardware and sensors which makes it more complex and costly. The Wiener filter based $\operatorname{method}^{7}$ does not need extra instruments; however, it needs to have the whole data simultaneously and therefore is not applicable in real time applications.

In contrast, the Kalman filter based approaches, such as the one presented by Izzetoglu et al.,$^{1}$ do not need extra sensors and also can perform motion artifact reduction in real time. In the Kalman filter based methods it is necessary to fit the input signal to a linear model such as an autoregressive (AR) model which was used by Izzetoglu et al., ${ }^{1}$ then the AR model should be transformed into state space representation. In the end, the Kalman filter is applied to estimate motionless data from motion corrupted data.

In this paper, we propose the autoregressive moving average (ARMA) model instead of the commonly used AR model, which has been used by Izzetoglu et al. ${ }^{1}$ before, to model the fNIRS motionless signal. Our results showed improved motion reduction, in terms of $\Delta \mathrm{SNR}$, compared to the AR model. We relate this improvement to the fact that ARMA model is richer in structure than AR. ARMA contains more terms than AR so that it will be fitted better than AR to any system. We evaluate the performance of AR and ARMA based methods by using real data simulated data in which a white Gaussian noise, instead of actual motion artifact, was added to a motionless signal. For simulated data, ARMA based method acted better than AR based method in noise effect reduction of the original signal in term of resulting in a higher $\triangle$ SNR. Our results show an improvement of about $2 \mathrm{~dB}$ in $\triangle \mathrm{SNR}$ for ARMA model over AR model after applying the ARMA-based Kalman filter to motion corrupted data in order to estimate motion corrected data.

The rest of the paper is organized as follows. In Sec. 2 the proposed algorithm is described in detail. The data set used in this work is introduced in that section as well. The results of the application of the proposed method to this data set as well as simulated data are brought in Sec. 3. Finally, Sec. 4 discusses and concludes the paper.

\section{Materials and Methods}

\section{1. fNIRS data}

The fNIRS data used in this work were recorded by a three channel fNIRS probe attached to the subject's forehead at cognitive neuroengineering and quantitative experimental research (CONQUER) CollabOrative at Drexel University (3508 Market Street, Philadelphia, PA 1904, Drexel University). fNIRS data were collected using a continuous wave fNIRS system. The fNIRS system is composed of three subsystems: (i) fNIRS sensors that consist of one light source and three photo detectors. The light source is a multi-wavelength light emitting diode (LED) manufactured by Epitex Inc. type 
L $4 * 730 / 4 * 850-40$ Q96-I. The LED comes in a STEM TO- 5 package at $730 \mathrm{~nm}$ and $850 \mathrm{~nm}$ wavelengths with an output power of 5 to $15 \mathrm{~mW}$. The photo detectors are manufactured by BurrBrown Corporation type OPT101 and come in an eight-pin DIP package. (ii) A control box for operating the LEDs and photo detectors. (iii) A desktop computer running the cognitive optical brain imaging (COBI) studio software developed in the laboratory for data acquisition and real-time data visualization. Three channels are used to record fNIRS signals. Source-detector distances for the channels are 2.8, 2.8 and $1 \mathrm{~cm}$. This system is the same that was used by Barati et al. in their recent work. ${ }^{12}$ Sampling frequency is $2 \mathrm{~Hz}$. Six healthy, right handed individuals (three males) with no history of neurological, psychological, or psychiatric disorders who were analgesic-free were recruited from the Drexel University community. All participants signed the informed consent form approved by the institutional review board (IRB) at Drexel University. Examples of the recorded signals are shown in Fig. 1.

\section{Protocol}

In this study we have concentrated on the motion artifact reduction in the functional NIRS signals. This artifact is generated due to the head motions of the subject. Figure 1 shows the motion corrupted oxy-Hb and deoxy-Hb in solid and dotted lines, respectively. The following protocol has been used.

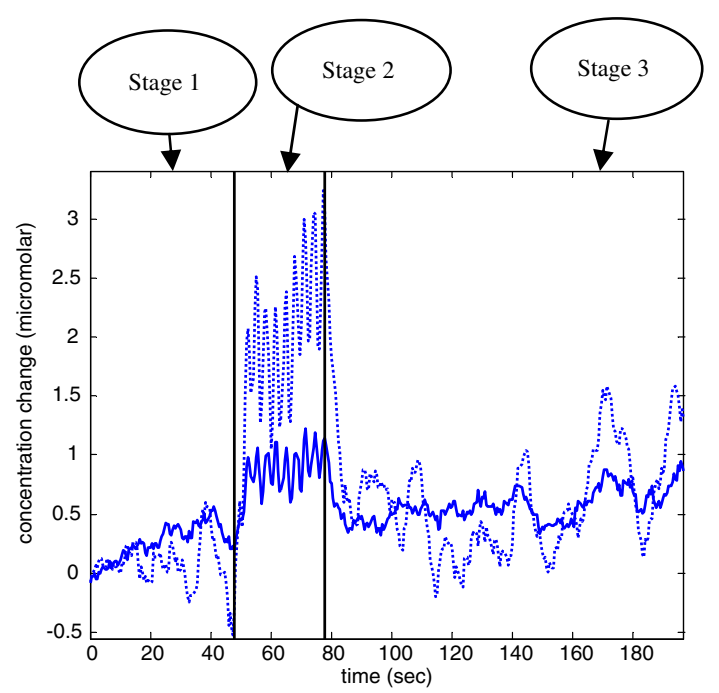

Fig. 1. Example of fNIR signals including three stages, stage 1 is rest, and then subject was moving his/her head during stage 2 , and stage 3 is post motion, solid line was used for deoxy-Hb and the dotted line for oxy-Hb.
The first stage includes one minute baseline signal in which the subject was instructed to sit still and relax (rest stage). This stage is considered as motionless signal. Then the subject was instructed to move his/her head up and down in a steady frequency around $0.3 \mathrm{~Hz}$ for $30 \mathrm{~s}$ (three movements in each ten seconds). This motion artifact is considered as slow head motion. The experiment ended with two minutes post recording with no motion (post motion stage).

As a result each of the two oxy-Hb and deoxy-Hb signals was contaminated by the motion artifacts.

Figure 2 shows a typical fNIRS signal including rest and head motion stages. The post motion stage is removed. Since then, we need just these two parts, rest and motion, and deal with such signals like to that presented in Fig. 2.

We first model the fNIRS signal using the commonly used AR model like to that was used by Izzetoglu et al. ${ }^{1}$ as well as ARMA model which is our proposed modification. The linear models then should be transformed into state space representations. Finally Kalman filter will be applied to motion corrupted functional NIRS data. We evaluate the ability of algorithm by applying it to some simulated data in which white Gaussian noise as artificial artifact is added to motionless signal. These noises are generated by MATLAB. We do not set the value of their variances initially, but just after generating by MATLAB we calculate them so

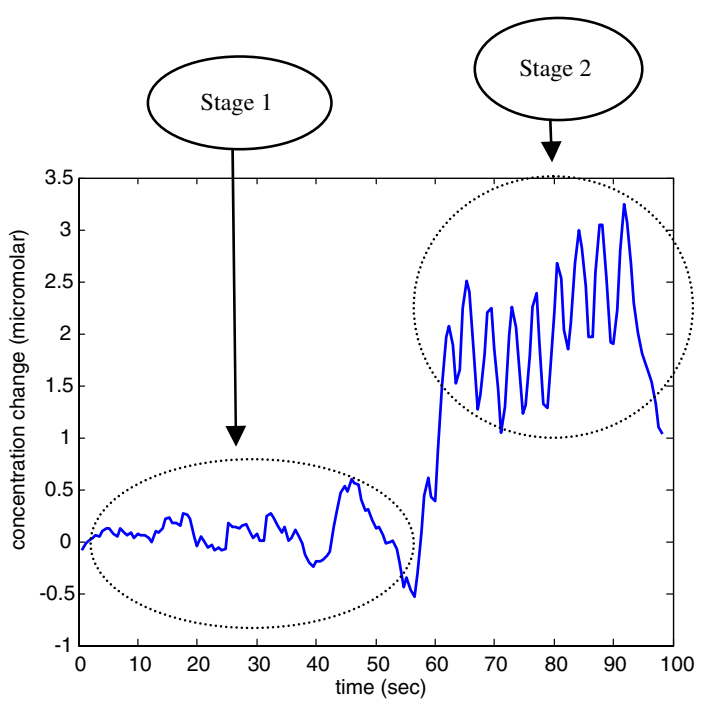

Fig. 2. Example of a typical fNIR signal with rest and head motion stages (stage 1 and stage 2 , respectively). 
that they will be known to us. Since the noises are random generated by MATLAB and we do not play any role in their creations some variances may be repeated. Such repeats stem from random nature of creation of the noises. Then, the variance of such noise is estimated through the algorithms explained before. We expect the algorithm to be able to estimate the variance of noise as close to the real variance value, which is known for us now, as possible. At the next step, the actual (real) data will be fed to the algorithm. In this way we obtain corrected fNIRS signals from the motion corrupted signals. $\Delta \mathrm{SNR}$ which has been used by Izzetoglu et al. ${ }^{1}$ is calculated as a quantitative measure for comparing the results.

\subsection{Linear model}

In most cases, it is likely that not the entire recorded data is contaminated by motion artifact. Usually, motion artifact corrupted parts of the signal are easily distinguishable from motionless parts. Sudden continuous high frequency oscillations are usually easy to distinguish. However, sometimes the need to detect motion artifact may be problematic to some extent so that it may be considered as a disadvantage of Kalman filtering method.

We will use motionless parts of NIRS data as well as motion corrupted parts to calculate required parameters. At first, the fNIRS motionless signal (stage 1 ) is modeled as an AR model. The optimal AR order for the system (fNIRS motionless signal) was obtained by Akaike information criterion (AIC) as $N=4$. An AR model of order of 4 , is as follows:

$$
x_{k}=a_{1} x_{k-1}+\cdots+a_{4} x_{k-4}+w_{k} .
$$

We can see from Eq. (1) how a sample of signal in the present time point $k$ is linearly related to its previous values and to the noise.

In Eq. (1), $w_{k}$ is a white Gaussian noise that its variance will be calculated later.

where $a_{i}, i=1, \ldots, 4$ are AR coefficients. We did not obtain the coefficients manually, but we used "ar" function in MATLAB to obtain them directly and automatically.

At the next stage, the linear model is transformed into the state space representation as below ${ }^{1}$;

$$
\begin{gathered}
\mathbf{x}_{\mathbf{k}}=A \mathbf{x}_{\mathbf{k}-\mathbf{1}}+\mathbf{w}_{\mathbf{k}}, \\
z_{k}=H \mathbf{x}_{\mathbf{k}}+v_{k},
\end{gathered}
$$

where

$$
\mathbf{x}_{\mathbf{k}}=\left[\begin{array}{c}
x_{k} \\
x_{k-1} \\
\vdots \\
x_{k-N+1}
\end{array}\right]_{N \times 1}, \quad \mathbf{w}_{\mathbf{k}}=\left[\begin{array}{c}
w_{k} \\
0 \\
\vdots \\
0
\end{array}\right]_{N \times 1} .
$$

Here $\mathbf{z}_{\mathbf{k}}$ is the motion corrupted signal vector, $\mathbf{x}_{\mathbf{k}}$ is the motionless signal vector, $\mathbf{w}_{k}$ is the system noise vector and $v_{k}$ is the measurement noise. In general, $A$ is an $\mathrm{N} \times \mathrm{N}$ matrix and $H$ is a $1 \times \mathrm{N}$ row vector as follows ${ }^{1}$ :

$$
A=\left[\begin{array}{cccccc}
-m_{N} & -m_{N-1} & -m_{N-2} & \ldots & -m_{2} & -m_{1} \\
1 & 0 & 0 & \ldots & 0 & 0 \\
0 & 1 & 0 & \ldots & 0 & 0 \\
\vdots & & & & & \\
0 & 0 & 0 & \ldots & 1 & 0
\end{array}\right]_{N \times N}
$$

and

$$
H=\left[\begin{array}{lllll}
1 & 0 & \ldots & 0 & 0
\end{array}\right]_{1 \times N} .
$$

As it was mentioned before, $N=4$ here.

To estimate the covariance of system's noise, we assume

$$
\hat{w}_{k+1}=z_{k+1}-G \mathbf{x}_{\mathbf{k}}
$$

where

$$
G=\left[-m_{1}-m_{2}-m_{3}-m_{4}\right] .
$$

Here $\mathbf{x}_{\mathbf{k}}$ is the AR sample vector and $z_{k}$ is the measured signal. Index $k$ contains those time points in which we have motionless signal. Eventually, variance of $\hat{w}_{k+1}$ is calculated and considered as an estimation of the system's noise. Similarly, to estimate the variance of the measuring noise or motion artifact we assume:

$$
\hat{v}_{k+1}=z_{k+1}-H \mathbf{x}_{\mathrm{k}}
$$

where

$H$ is the same as that it was brought in (8).

In Eq. (9) index $k$ includes those time points during which head motions of the subject exist.

We assume the statistics of the system and the measurement noise to be $w_{k}$ and $v_{k}$, respectively, and also they are independent of each other, with 
white Gaussian distributions $\mathbf{w}_{\mathbf{k}} \sim N(0, Q), \mathbf{v}_{\mathbf{k}} \sim$ $N(0, R) .{ }^{1}$ Kalman filtering equations are as follows ${ }^{1}$ :

- Time update equations:

$$
\begin{gathered}
\hat{\mathbf{x}}_{\mathbf{k}}^{-}=\mathrm{A} \hat{\mathbf{x}}_{\mathbf{k}-1}^{-}, \\
P_{k}^{-}=A P_{k-1} A^{T}+Q .
\end{gathered}
$$

- Measurement update equations:

$$
\begin{gathered}
K_{k}=P_{k}^{-} H^{T}\left(H P_{k}^{-} H^{T}+R\right)^{-1}, \\
\hat{\mathbf{x}}_{\mathbf{k}}=\hat{\mathbf{x}}_{\mathbf{k}}^{-}+K_{k}\left(z_{k}-H \hat{\mathbf{x}}_{\mathbf{k}}^{-}\right), \\
P_{k}=\left(I-K_{k} H\right) P_{k}^{-} .
\end{gathered}
$$

In which $P_{k}^{-}$is a priori covariance matrix (error covariance matrix before update) as follows ${ }^{1}$ :

$$
\begin{gathered}
\mathbf{e}_{\mathbf{k}}^{-}=\mathbf{x}_{\mathbf{k}}-\hat{\mathbf{x}}_{\mathbf{k}}^{-}, \quad P_{k}^{-}=E\left[\mathbf{e}_{\mathbf{k}}^{-} \mathbf{e}_{\mathbf{k}}^{-T}\right], \\
\mathbf{e}_{\mathbf{k}}=\mathbf{x}_{\mathbf{k}}-\hat{\mathbf{x}}_{\mathbf{k}}, \quad P_{k}=E\left[\mathbf{e}_{\mathbf{k}} \mathbf{e}_{\mathbf{k}}^{T}\right],
\end{gathered}
$$

where $K_{k}$ is Kalman gain matrix. ${ }^{11}$

A quantitative criterion, $\Delta \mathrm{SNR}$, as has been used by Izzeotglu et al. ${ }^{1}$ is as follows:

$$
\Delta \mathrm{SNR}=\mathrm{SNR}_{e}-\mathrm{SNR}_{i} .
$$

In Eq. (17), $\mathrm{SNR}_{e}$ is the estimation signal to noise ratio and $\mathrm{SNR}_{i}$ is the input signal to noise ratio. Their definitions are as follows ${ }^{1}$ :

$$
\mathrm{SNR}_{e}=10 \log \left(\frac{\sigma_{x}^{2}}{\sigma_{e}^{2}}\right) .
$$

In Eq. (18), $\sigma_{x}^{2}$ is variance of the motionless signal and $\sigma_{e}^{2}$ is the variance of estimation error $e(n)$.

$$
e(n)=x(n)-\hat{x}(n) .
$$

The estimation error, as we see from Eq. (19), is the difference between the motionless fNIRS signal and the corrected signal after applying Kalman filtering method. ${ }^{1}$

$$
\mathrm{SNR}_{i}=10 \log \left(\frac{\sigma_{x}^{2}}{\sigma_{v}^{2}}\right) .
$$

In Eq. (20), $\sigma_{v}^{2}$ is the variance of the motion artifact. ${ }^{1}$

Now we develop an ARMA model that is a richer model in structure than AR, for the motionless fNIRS data. Optimal order of model is obtained using AIC as $(4,4)$. The parameters of model directly and automatically were estimated using "armax" function in MATLAB. The model that MATLAB assumes for ARMA is as follows:

$$
N(q) y(n)=C(q) e(n)
$$

where

$$
N(q)=1+n_{1} q^{-1}+n_{2} q^{-2}+n_{3} q^{-3}+n_{4} q^{-4}
$$

and

$$
C(q)=1+c_{1} q^{-1}+c_{2} q^{-2}+c_{3} q^{-3}+c_{4} q^{-4} .
$$

According to MATLAB help notes, for estimating $n_{i}$ and $c_{i}$ the software uses a recursive approach through which minimizes estimation error. Also, the cost function is the determinant of input covariance matrix.

Then this linear model should be converted into a space state representation. There are numerous state space representations for a linear system, however we examined some of them and chose the following representation that resulted in better outcome in terms of $\Delta$ SNR as well as an acceptable appearance of the estimated motionless signal:

$$
\begin{gathered}
\mathbf{x}_{\mathbf{k}}=F \mathbf{x}_{\mathbf{k}-1}+B w_{k}, \\
z_{k}=H \mathbf{x}_{\mathbf{k}}+v_{k},
\end{gathered}
$$

where

$$
\begin{gathered}
B=\left[\begin{array}{l}
-n_{1}+c_{1} \\
-n_{2}+c_{2} \\
-n_{3}+c_{3} \\
-n_{4}+c_{4}
\end{array}\right], \\
F=\left[\begin{array}{llll}
-n_{1} & 1 & 0 & 0 \\
-n_{2} & 0 & 1 & 0 \\
-n_{3} & 0 & 0 & 1 \\
-n_{4} & 0 & 0 & 0
\end{array}\right] .
\end{gathered}
$$

\subsubsection{Estimation of variance of the system's noise}

At this step the variance of the system's noise, $w_{k}$, should be estimated. ARMA model can be written as:

$$
\begin{aligned}
y(k)= & -n_{1} y(k-1)-\cdots-n_{4} y(k-4)+e(k) \\
& +c_{1} e(k-1)+\cdots+c_{4} e(k-4) .
\end{aligned}
$$

We can rewrite Eq. (28) as

$$
\begin{gathered}
e(k)=y(k)+n_{1} y(k-1)+\cdots+n_{4} y(k-4) \\
-c_{1} e(k-1)-\cdots-c_{4} e(k-4) .
\end{gathered}
$$

One can obtain value of noise iteratively from Eq. (29) by knowing the initial value of the noise. We assume the initial values of noise as zero. After estimating the noise in this way, the variance of the noise can be calculated. 


\subsubsection{Estimation of variance of the measurement noise}

To estimate the variance of the measurement noise, $v_{k}$, which is regarded as the motion artifact, the procedure is as following. An estimation of measurement noise can be as follows:

$$
\hat{v}_{k+1}=z_{k+1}-H \mathbf{x}_{\mathbf{k}} .
$$

Whenever $\hat{v}_{k}$ is estimated, the variance of measurement noise can be calculated.

By now we gathered all requirements for performing Kalman filter. Now, we can feed a motion corrupted signal, which its characteristics has been derived through our previous computations that we elaborated above, to the Kalman filter in order to obtain motionless signal as the output of the filter. Both simulated and real (actual) data are fed to Kalman filter. The results of their outputs will be presented in the next section.

\section{Results}

\subsection{Simulated data}

To evaluate the accuracy of the method, both AR and ARMA based algorithms are applied to the simulated data.

Results of applying AR based algorithm to simulated data shown in Table 1.

In Fig. 3(a) an example of a white and Gaussian noise is shown. Such noise added to an fNIRS signal (stage 1) and the resulted signal is shown in (b). and in (c) the cleaned signal by AR based method is printed in dashed-line and the original signal from stage 1 in solid line.

The result of testing algorithm relating to ARMA based method is shown in Table 2 .

Table 1. Results of applying AR based method to simulated data.

\begin{tabular}{cccc}
\hline $\begin{array}{l}\text { Trail } \\
\text { no. }\end{array}$ & $\begin{array}{c}\text { Covariance of } \\
\text { Gaussian noise }\end{array}$ & $\begin{array}{c}\text { Estimated covariance } \\
\text { of noise }\end{array}$ & $\Delta$ SNR \\
\hline 1 & 0.10 & 0.12 & 3.21 \\
2 & 0.07 & 0.08 & 1.63 \\
3 & 0.08 & 0.10 & 2.63 \\
4 & 0.09 & 0.10 & 2.45 \\
5 & 0.07 & 0.08 & 1.82 \\
6 & 0.12 & 0.14 & 2.83 \\
7 & 0.10 & 0.14 & 3.70 \\
\hline
\end{tabular}

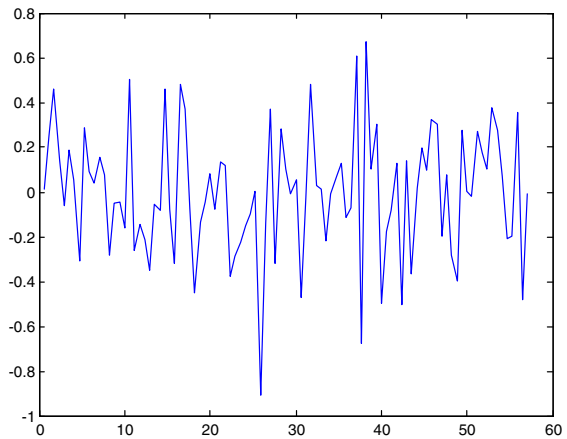

(a)

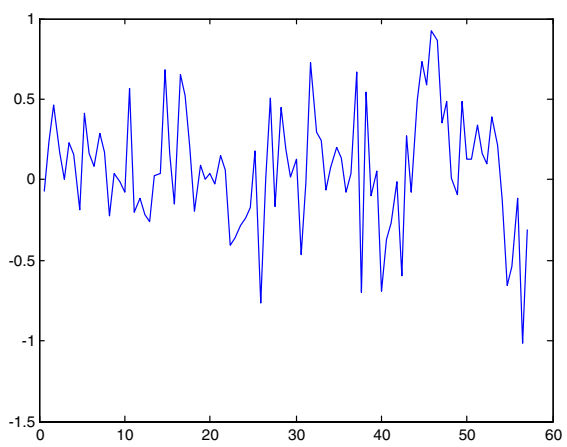

(b)

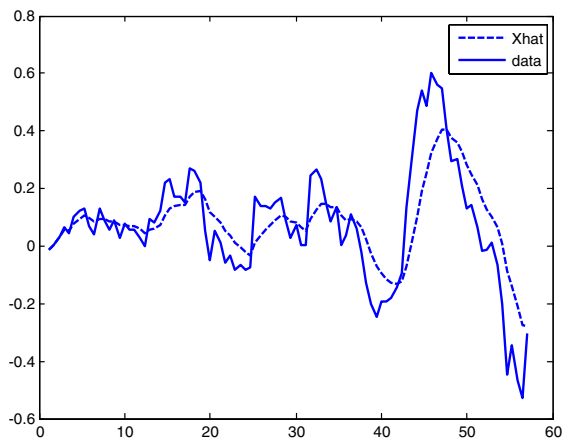

(c)

Fig. 3. (a) A typical pattern of an artificial noise (we assume as white and Gaussian), (b) The noise in (a) added to an fNIRS signal, (c) The contaminated signal in (b) is cleaned by AR based method (dashed line) and the original motionless signal (solid line).

For simulated data, the average of $\Delta \mathrm{SNR}$ values in Table 1 (AR approach) is $2.61 \mathrm{~dB}$, and is $6.26 \mathrm{~dB}$ in Table 2 (ARMA approach).

In Fig. 4(a) we demonstrated another white and Gaussian noise. Such noise added to a motionless fNIRS signal (stage 1) and the corrupted is shown in (b). In (c) the cleaned signal by the ARMA model and the original motionless signal are shown. 
Table 2. Results of applying ARMA based method to simulated data.

\begin{tabular}{cccc}
\hline $\begin{array}{l}\text { Trail } \\
\text { no. }\end{array}$ & $\begin{array}{c}\text { Covariance of } \\
\text { Gaussian noise }\end{array}$ & $\begin{array}{c}\text { Estimated covariance } \\
\text { of noise }\end{array}$ & $\Delta$ SNR \\
\hline 1 & 0.08 & 0.14 & 5.02 \\
2 & 0.08 & 0.17 & 5.68 \\
3 & 0.10 & 0.19 & 6.29 \\
4 & 0.11 & 0.23 & 7.11 \\
5 & 0.12 & 0.22 & 7 \\
6 & 0.10 & 0.19 & 6.33 \\
7 & 0.08 & 0.19 & 6.4 \\
\hline
\end{tabular}

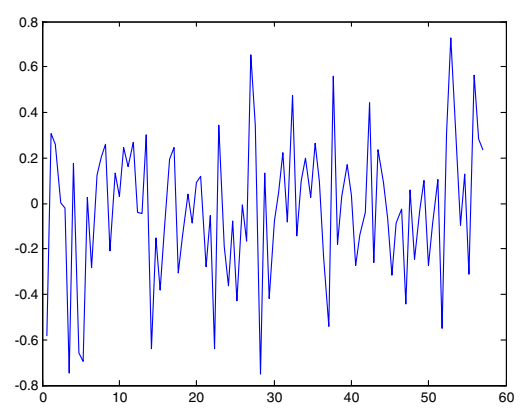

(a)

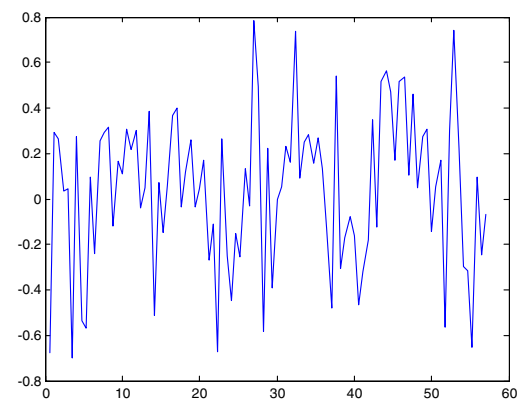

(b)

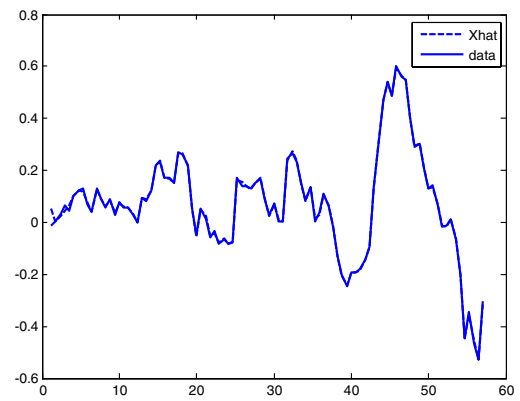

(c)

Fig. 4. A typical pattern of an artificial noise (we assume as white and Gaussian), (b) The noise in (a) added to an fNIRS signal, (c) The contaminated signal in (b) is cleaned by ARMA based method (dashed line) and the original motionless signal (solid line).
Table 3. Results of applying AR based method to real data.

\begin{tabular}{ccccccc}
\hline Signal index & 1 & 2 & 3 & 4 & 5 & 6 \\
\hline$\Delta \mathrm{SNR}$ & 10.39 & 6.22 & 9.08 & 7.70 & 10 & 8.94 \\
\hline
\end{tabular}

\subsection{Real (actual) data}

In this part we use the algorithms to estimate clear data from motion corrupted real NIR signals. Table 3 shows the calculated values of $\Delta$ SNR for several signals in AR method.

Figure 5 demonstrates one of the actual motion corrupted fNIRS signals (stage 2) and its clear estimated one by AR based method.

The horizontal axis of Fig. 5 is not the same as in Figs. 3 and 4 so that this issue may seem confusing. Notice that in Figs. 3 and 4 we plotted motionless part of signal (stage 1) while in Fig. 5 (and also in Fig. 6 later) we plot the motion part of the signal (stage 2). Therefore, the horizontal axis of Fig. 5 (and also 6) should be different from those of Figs. 3 and 4.

The quantitative results due to computing through ARMA based method brought in Table 4.

For real data, the average of $\triangle \mathrm{SNR}$ values in Table 3 (AR approach) is $8.7 \mathrm{~dB}$, and is $10.4 \mathrm{~dB}$ in Table 4 (ARMA approach).

An example of the motion artifact reduction of a contaminated real signal in ARMA method, is shown in Fig. 6 where the contaminated signal drawn as solid line and the cleared on as dashed line.

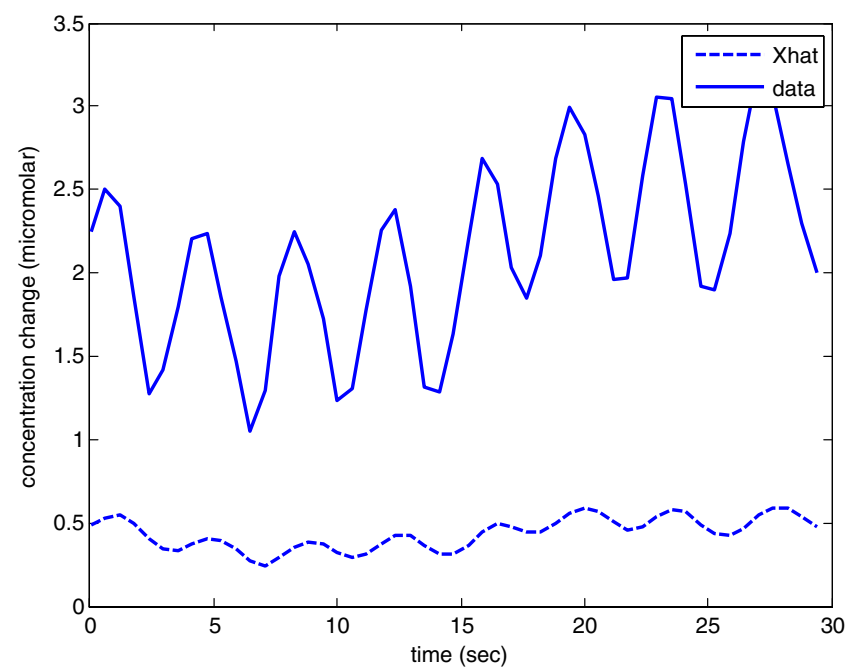

Fig. 5. An actual experimental contaminated signal (solid line) from stage 2 where the subject was moving his/her head and denoised signal (dashed line) after using AR based method. 


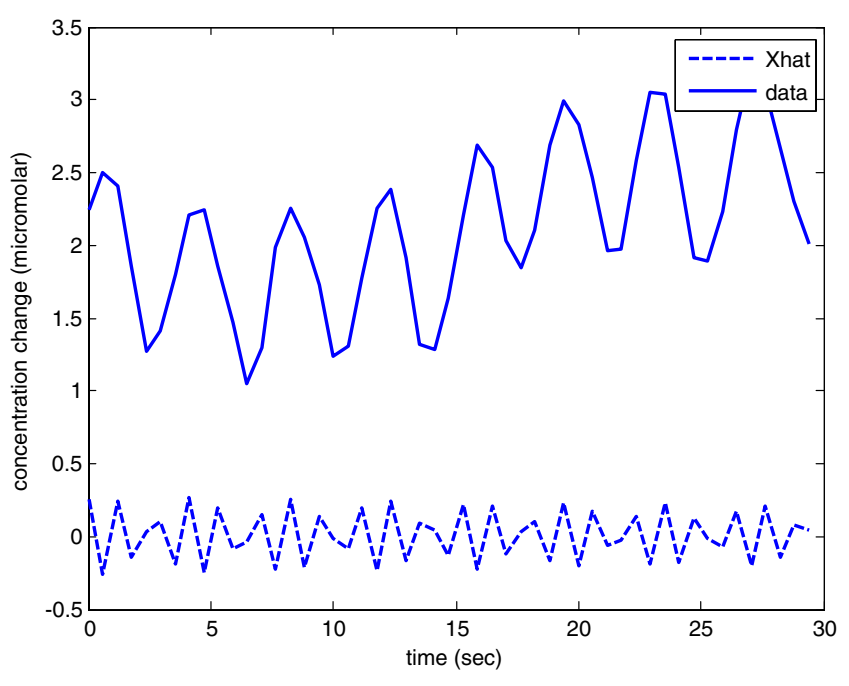

Fig. 6. Real contaminated signal (solid line) and denoised signal (dashed line) after using ARMA based method.

Table 4. Results of applying ARMA based method to real data.

\begin{tabular}{ccccccc}
\hline Signal index & 1 & 2 & 3 & 4 & 5 & 6 \\
\hline$\Delta \mathrm{SNR}$ & 9.48 & 13.24 & 9.27 & 9.34 & 9.92 & 10.96 \\
\hline
\end{tabular}

\section{Discussion}

In the previous section, the result of applying $\mathrm{AR}$ based method, which has been introduced previously by Izzatoglu et al. ${ }^{1}$ as well as ARMA based method, which we proposed, presented. The cleared signals of both simulated data and real data in ARMA based approach have higher quality in comparison to $\mathrm{AR}$ in term of $\triangle \mathrm{SNR}$ as a quantitative measure. As we see from Eqs. (3), (24) and (25), ARMA model includes more terms rather than AR. Therefore, ARMA can be better fitted to any system.

As we saw from Tables 1 and 2, associated to simulated data, both algorithms are successful in estimating the white Gaussian noise variances. According to average values which we presented in the previous section, $\triangle \mathrm{SNR}$, as the quantitative criterion, grows in average more than $3 \mathrm{~dB}$ in ARMA results. From signal appearance point of view, as Fig. 4(c) shows, for simulated data, the ARMA based method was very successful in estimating motionless data from motion corrupted data. Notice that in Fig. 4(c) there are really two signals, the corrected signal in dashed line and the original motionless signal in solid line; but because of precise estimation and good performance of algorithm on simulated data someone may virtually distinguish just one signal. By now, we assessed the performance of ARMA over AR and proved, at least for simulated data, that ARMA based method acted better than AR based method in estimating motionless signal from motion corrupted signal in term of $\Delta$ SNR. However, some real data are also needed to evaluate the performance in real case.

According to the average values which we presented in previous section, the quantitative measure, $\Delta \mathrm{SNR}$, demonstrates an average improvement of about $2 \mathrm{~dB}$ in ARMA results. The results of real data verify those of simulated data and both kind of results endorse that ARMA based method which we proposed acted better than AR based method which previously had been introduced by Izzetoglu et al. ${ }^{1}$ in motion artifact reduction of fNIRS signal.

\section{Acknowledgment}

We highly appreciate Professor Kambiz Pourrezaei and Dr. Zeinab Barati, PhD, from Drexel University, Philadelphia, PA, who provided us with the data used in this work. Our grateful thanks go to them for their useful and helpful guide to complete our work.

\section{References}

1. M. Izzetoglu, P. Chitrapu, S. Bunce, B. Onaral, "Motion artifact cancellation in NIR spectroscopy using discrete Kalman filtering," Biomed. Eng. 9, 1-10 (2010).

2. F. Mattehews, B. A. Pearlmutter, T. E. Wrad, C. Soraghan, C. Markham, "Hemodynamics for Brain-Computer Interfaces," IEEE Signal Process. Mag. 25, 87-94 (2008).

3. J. Gervain, J. Mehler, J. F. Werker, C. A. Nelson, G. Csibra, S. Lioyd-Fox, M. Shukla, R. N. Aslin, "Near-infrared spectroscopy: A report from the McDonnell infant methodology consortium," Dev. Cogn. Neurosci. 1, 22-46 (2011).

4. M. Cope, "The application of near infrared spectroscopy to noninvasive monitoring of cerebral oxygenation in the newborn infant," Ph.D. dissertion, Department of Medical Physics Bioengineering, College London, London, England, 1991.

5. F. F. Jobsis, "Noninvasive infrared monitoring of cerebral and myocardial oxygen sufficiency and circulatory parameters," Science 198, 1264-1267 (1977). 
6. M. Izzetoglu, A. Devaraj, K. Izzetoglu, S. Bunce, B. Onaral, "Motion artifact removal in fNIR signals using adaptive filtering," Proc. EMBS, Nashville, TN, 2003.

7. M. Izzetoglu, A. Devaraj, S. Bunce, B. Onaral, "Motion artifact cancellation in NIR spectroscopy using wiener filtering," IEEE Trans. Biomed. Eng. $52(2005)$.

8. X. Sui, S. Bray, A. L. Reiss, "Functional near infrared spectroscopy (NIRS) signal improvement based on negative correlation between oxygenated and deoxygenated hemoglobin dynamics," NeuroImage 49, 3039-3046 (2010).

9. F. Scholkmann, S. Spichtig, T. Muehlemann, M. Wolf, "How to detect and reduce movement artifacts in near-infrared imaging using moving standard deviation and spline interpolation," Physiol. Measure. 31 (2010).

10. B. Molavi, G. Dumont, B. Shadgan, "Motion artifact removal from muscle NIR spectroscopy measurements," IEEE Canadian Conf. Electrical and Computer Engineering, Calgary, Alberta, Canada, 2010.

11. M. S. Grewal, A. P. Andrews, Kalman Filtering: Theory and Practice Using MATLAB, 2nd edition, John Wiley \& Sons Inc. (2001).

12. Z. Barati, P. M. Shewokis, M. Izzetoglu, R. Polikar, G. Mychaskiw, K. Pourrezaei, "Hemodynamic response to repeated noxious cold pressor tests measured by functional near infrared spectroscopy on forehead," Ann. Biomed. Eng. 41 (2012). 